\title{
MONOTONIC FUNCTIONS RELATED TO THE $q$-GAMMA AND $q$-TRIGAMMA FUNCTIONS WITH APPLICATIONS
}

\author{
KHALED MEHREZ
}

Abstract. In this paper our aim is to investigate necessary and sufficient conditions for the complete monotonicity properties of some functions related to the $q$-gamma and $q$-trigamma functions. As application of this results, some new inequalities are derived. Our results are shown to be as a generalization of results which were obtained by Qi [6].

Mathematics subject classification (2010): 33D05, 26D07, 26A48.

Keywords and phrases: Completely monotonic functions, $q$-gamma function, $q$-trigamma function, inequalities.

\section{REFERENCES}

[1] C. H. KimberLing, A probabilistic interpretation of complete monotonicity, Aequationes Math. 10, (1974), 152-164.

[2] K. Mehrez, A Class of logarithmically completely monotonic functions related to the q-gamma function and applications, Positivity, (2016).

[3] D. S. MоAK, The q-analogue of Stirling's formula, Rocky Mountain J. Math. 14, (1984), 403-412.

[4] A. SALEM, A completely monotonic function involving q-gamma and q-digamma functions, J. Appr Theory, 164, (2012), 971-980.

[5] R. L. Schilling, R. Song, Z. Vondracek, Bernstein Functions. Theory and Applications, Studies in Mathematics, vol. 37, Walter de Gruyter, Berlin (2010).

[6] F. QI, A completely monotonic function involving the gamma and trigamma functions, Kuwait Journal of Science (2016), in press. 